\title{
Estimating Effectiveness of Some Methods for Detecting Edge of Potential Field Sources
}

\author{
Le Thi Sang ${ }^{1}$, Vu Duc Minh ${ }^{1}$, Lai Manh Giau², Ngo Thi To Nhu ${ }^{1}$, \\ Nguyen Xuan Tuyen ${ }^{3}$, Do Duc Thanh ${ }^{1}$, Pham Thanh Luan ${ }^{1, *}$ \\ ${ }^{1}$ VNU University of Science, 334 Nguyen Trai, Hanoi, Vietnam \\ ${ }^{2}$ Union of Geophysics, 95 Chien Thang, Ha Dong, Hanoi, Vietnam \\ ${ }^{3}$ University of Transport and Communications, 3 Cau Giay, Dong Da, Hanoi, Vietnam
}

Received 09 October 2020

Revised 27 November 2020; Accepted 30 November 2020

\begin{abstract}
This paper presents a comparative study of effectiveness of edge detection methods such as total horizontal gradient, analytic signal amplitude, tilt angle, gradient amplitude of tilt angle, theta map, horizontal tilt angle, tilt angle of total horizontal gradient, tilt angle of analytic signal, improved theta map, and total horizontal gradient of improved tilt angle. The effectiveness of each method was estimated on synthetic magnetic data and synthetic gravity anomaly data with and without noise. The obtained results show that the tilt angle of gradient amplitude can detect all the edges more clearly and precisely. The applicability of each method is demonstrated on the aeromagnetic anomaly data from the Zhurihe region of Northeast China, and Bouguer gravity anomaly data from a region of North Vietnam. The results computed by the tilt angle of horizontal gradient were also in accord with the geologic structures of the areas.
\end{abstract}

Keywords: Edge detection, magnetic anomaly, gravity anomaly, Zhurihe, North Vietnam.

\footnotetext{
* Tác giả liên hệ.

Địa chi email: luanpt@ hus.edu.vn

https://doi.org/10.25073/2588-1140/vnunst.5140
} 


\title{
Đánh giá hiệu quả một số phương pháp xác định biên của nguồn gây dị thường trường thế
}

\author{
Lê Thị Sang ${ }^{1}$, Vũ Đức Minh ${ }^{1}$, Lại Mạnh Giàu², Ngô Thị Tố Như ${ }^{1}$, \\ Nguyễn Xuân Tuyên ${ }^{3}$, Đỗ Đức Thanh ${ }^{1}$, Phạm Thành Luân ${ }^{1, *}$ \\ ${ }^{1}$ Truờng Đại học Khoa học Tự nhiên, Đại học Quốc gia Hà Nội, \\ 334 Nguyễn Trãi, Hà Nội, Việt Nam \\ ${ }^{2}$ Liên đoàn Vật lý Địa chất, số 1, ngõ 95, Chiến Thắng, Văn Quán, Hà Đông, Hà Nội, Việt Nam \\ ${ }^{3}$ Truò̀ng Đại học Giao thông Vận tải, số 3 Cầu Giấy, Đống Đa, Hà Nội, Việt Nam. \\ Nhận ngày 09 tháng 10 năm 2020 \\ Chỉnh sửa ngày 27 tháng 11 năm 2020; Chấp nhận đăng ngày 30 tháng 11 năm 2020
}

\begin{abstract}
Tóm tắt: Xác định biên ngang của nguồn gây dị thường là một nhiệm vụ quan trọng trong xử lý, phân tích tài liệu trường thế. Một loạt các phương pháp xác định biên ngang đã được phát triển dựa trên các đạo hàm ngang và thẳng đứng của dị thường trường thế. Bài báo này trình bày một nghiên cứu so sánh về hiệu quả của các phương pháp xác định biên như phương pháp gradient ngang toàn phần, biên độ tín hiệu giải tích, góc nghiêng, gradient ngang của góc nghiêng, bản đồ theta, góc nghiêng ngang, góc nghiêng của gradient ngang toàn phần, góc nghiêng của biên độ tín hiệu giải tích, bản đồ theta cải tiến, gradient ngang toàn phần của góc nghiêng cải tiến. Hiệu quả của các phương pháp được đánh giá trên mô hình từ và mô hình trọng lực chứa nhiễu và không chứa nhiễu. Kết quả thu được chỉ ra rằng, phương pháp góc nghiêng của gradient ngang toàn phần có thể xác định chính xác và đầy đủ các ranh giới ngang của nguồn. Khả năng áp dụng thực tế của các phương pháp được đánh giá thông qua phân tích bản đồ dị thường từ hàng không khu vực Zhurihe, Đông Bắc Trung Quốc và bản đồ trọng lực Bouguer từ một khu vực thuộc miền Bắc Việt Nam. Các kết quả thu được từ phương pháp góc nghiêng của gradient ngang toàn phần phù hợp với cấu trúc địa chất của các khu vực nghiên cứu.
\end{abstract}

Tù khóa: Xác định biên, dị thường từ, dị thường trọng lực, Zhurihe, Bắc Việt Nam.

\section{Mở đầu}

Hiểu biết về vị trí biên của nguồn gây dị thường trường thế rất quan trọng trong việc lập bản đồ địa chất cũng như các ứng dụng môi trường và kỹ thuật $[1,2]$. Tuy nhiên, do hình dáng của trường quan sát phụ thuộc vào nhiều tham số nên các ranh giới ngang nguồn khó có thể xác định trực tiếp từ bản đồ trường [3,4]. Để giải quyết vấn đề đó, một loạt các phương pháp xác định biên đã được phát triển. Các phương pháp này được xây dựng dựa trên các đạo hàm ngang hoặc đạo hàm thẳng đứng của dị thường trường thế hoặc dựa trên sự kết hợp giữa các đạo hàm đó [5-9]. Thông thường, các phương pháp xác định biên được chia thành hai nhóm chính, gồm: nhóm phương pháp dựa trên biên độ đạo hàm và nhóm phương pháp dựa trên pha. Evjen (1936), Cordell và Grauch (1985), Roest và nnk (1992), Hsu và nnk (1996), Fedi và Florio (2001), Verduzco và nnk (2004), Cella và nnk (2009), Beiki (2010) đã phát triển các phương pháp dựa

\footnotetext{
* Tác giả liên hệ.

Địa chi email: luanpt@hus.edu.vn

https://doi.org/10.25073/2588-1140/vnunst.5140
} 
trên biên độ đạo hàm để làm nổi bật ranh giới ngang của các cấu trúc từ tính và mật độ $[1,2,10$ 15]. Để xác định đồng thời biên ngang của các cấu trúc nằm ở những độ sâu khác nhau, Miller và Singh (1994), Wijns và nnk (2005), Cooper và Cowan (2006), Ferreira và nnk (2013), Yuan và Yu (2014), Cooper (2014), Chen và nnk (2017), Nasuti và nnk (2018), Pham và nnk (2018a, b, 2019a, b, 2020b) đã phát triển các phương pháp pha dựa trên tỷ số của các đạo hàm [3,16-28]. Ưu điểm của các phương pháp này là không yêu cầu biết trước các thông tin về nguồn gây dị thường.

Trong nghiên cứu này, chúng tôi sẽ đánh giá hiệu quả của các phương pháp xác định biên được sử dụng phổ biến và một số phương pháp được phát triển gần đây. Các phương pháp đó bao gồm: gradient ngang toàn phần THG [11], biên độ tín hiệu giải tích AS [12], góc nghiêng TA [16], gradient ngang của góc nghiêng THG_TA [13], bản đồ theta TM [17], góc nghiêng ngang TDX [18], góc nghiêng của gradient ngang toàn phần [3], góc nghiêng của biên độ tín hiệu giải tích TAS [20], bản đồ theta cải tiến ITM [21], gradient ngang toàn phần của góc nghiêng cải tiến THG_STDR [22]. Khả năng áp dụng của các phương pháp được đánh giá thông qua phân tích các mô hình cũng như tài liệu từ hàng không khu vực Zhurihe, Trung Quốc và tài liệu trọng lực miền Bắc Việt Nam.

\section{Các phương pháp xác định biên}

Trong phần này, chúng tôi sẽ trình bày ngắn gọn cơ sở lý thuyết của các phương pháp xác định biên.

Phương pháp gradient ngang toàn phần được giới thiệu bởi Cordell và Grauch (1985) là một trong những phương pháp xác định biên được sử dụng phổ biến cho tới tận ngày nay. Phương pháp được cho bởi biểu thức [11]:

$$
T H G=\sqrt{\left(\frac{\partial F}{\partial x}\right)^{2}+\left(\frac{\partial F}{\partial y}\right)^{2}}
$$

trong đó $(\partial \mathrm{F} / \partial \mathrm{x})$ và $(\partial \mathrm{F} / \partial \mathrm{y})$ lần lượt là các đạo hàm của dị thường trường thế $F$ theo các hướng $\mathrm{x}, \mathrm{y}$. Phương pháp sinh ra các giá trị cực đại trên biên của nguồn. Đây được coi là cách tiếp cận đơn giản nhất để xác định ranh giới ngang của các cấu trúc mật độ hoặc từ tính.

Một phương pháp phổ biến khác, gọi là biên độ tín hiệu giải tích (hoặc tổng gradient), được giới thiệu bởi Roest và nnk (1992). Các giá trị của biên độ tín hiệu giải tích được xác định như sau [12]:

$$
A S=\sqrt{\left(\frac{\partial F}{\partial x}\right)^{2}+\left(\frac{\partial F}{\partial y}\right)^{2}+\left(\frac{\partial F}{\partial z}\right)^{2}}
$$

trong đó $(\partial \mathrm{F} / \partial \mathrm{z})$ là đạo hàm thẳng đứng của dị thường trường thế $F$. Biên độ tín hiệu giải tích cũng cung cấp các giá trị cực đại trên biên của nguồn. Do sử dụng biên độ đạo hàm nên hai phương pháp kể trên không thể cân bằng các dị thường gây bởi các nguồn nằm ở những độ sâu khác nhau.

Để cân bằng các dị thường có biên độ khác nhau, một loạt các phương pháp pha đã được phát triển. Các phương pháp này dựa trên việc chuẩn hóa các đạo hàm của dị thường trường thế. Một trong những phương pháp cân bằng đầu tiên là phương pháp góc nghiêng, được đề xuất bởi Miller và Singh (1994). Phương pháp thực hiện việc chuẩn hóa đạo hàm thẳng đứng bởi hàm gradient toàn phần. Phương pháp được thể hiện bằng biểu thức [16]:

$$
\mathrm{TA}=\operatorname{atan} \frac{\frac{\partial \mathrm{F}}{\partial \mathrm{z}}}{\sqrt{\left(\frac{\partial \mathrm{F}}{\partial \mathrm{x}}\right)^{2}+\left(\frac{\partial \mathrm{F}}{\partial \mathrm{y}}\right)^{2}}}
$$

Hàm TA đạt cực đại trên các nguồn với mật độ dư dương hoặc các nguồn từ có cường độ từ hóa dương và đạt cực tiểu trên các nguồn với mật độ dư âm hoặc các nguồn từ có cường độ từ hóa âm. Các đường đồng mức "không" trên bản đồ TA được sử dụng để xác định các ranh giới ngang của nguồn.

Verduzco và nnk (2004) đã tính toán gradient ngang toàn phần của góc nghiêng và chỉ ra rằng cách tiếp cận này hiệu quả hơn sử dụng 
góc nghiêng. Các giá trị của gradient ngang toàn phần của góc nghiêng được xác định như sau [13]:

$$
T H G_{-} T A=\sqrt{\left(\frac{\partial T A}{\partial x}\right)^{2}+\left(\frac{\partial T A}{\partial y}\right)^{2}}
$$

Hàm THG_TA sinh ra các giá trị cực đại trên ranh giới ngang của các cấu trúc mật độ hoặc từ tính.

Wijns và cộng sự (2005) giới thiệu một phương pháp pha khác, được gọi là phương pháp bản đồ theta. Phương pháp sử dụng biên độ tín hiệu giải tích để chuẩn hóa gradient ngang toàn phần. Phương pháp được cho bởi biểu thức [17]:

$$
T M=\operatorname{acos} \frac{\sqrt{\left(\frac{\partial \mathrm{F}}{\partial \mathrm{x}}\right)^{2}+\left(\frac{\partial \mathrm{F}}{\partial \mathrm{y}}\right)^{2}}}{\sqrt{\left(\frac{\partial \mathrm{F}}{\partial \mathrm{x}}\right)^{2}+\left(\frac{\partial \mathrm{F}}{\partial \mathrm{y}}\right)^{2}+\left(\frac{\partial \mathrm{F}}{\partial \mathrm{z}}\right)^{2}}}
$$

Khác với các phương pháp khác, phương pháp bản đồ theta đạt cực tiểu trên các ranh giới ngang của nguồn.

Cooper và Cowan (2006) đề nghị sử dụng một phương pháp sửa đổi của góc nghiêng, được gọi là phương pháp góc nghiêng ngang. Phương pháp sử dụng giá trị tuyệt đối của đạo hàm thẳng đứng để chuẩn hóa gradient ngang toàn phần và được định nghĩa bởi biểu thức [18]:

$$
\operatorname{TDX}=\operatorname{atan} \frac{\sqrt{\left(\frac{\partial \mathrm{F}}{\partial \mathrm{x}}\right)^{2}+\left(\frac{\partial \mathrm{F}}{\partial \mathrm{y}}\right)^{2}}}{\left|\frac{\partial \mathrm{F}}{\partial \mathrm{z}}\right|}
$$

Phương pháp góc nghiêng ngang đạt cực đại trên các ranh giới ngang của nguồn gây dị thường trường thế.

Ferreira và nnk (2013) đã thay thế biểu thức trong phương trình (3) bởi tỉ lệ các đạo hàm của gradient ngang toàn phần. Phương pháp sau đó được biết như phương pháp góc nghiêng của gradient ngang toàn phần. Phương pháp sử dụng gradient ngang toàn phần của $\mathrm{THG}$ để chuẩn hóa đạo hàm thẳng đứng của THG. Biểu thức toán học của phương pháp được cho bởi [3]:

$$
\mathrm{TTHG}=\operatorname{atan} \frac{\frac{\partial \mathrm{THG}}{\partial \mathrm{z}}}{\sqrt{\left(\frac{\partial \mathrm{THG}}{\partial \mathrm{x}}\right)^{2}+\left(\frac{\partial \mathrm{THG}}{\partial \mathrm{y}}\right)^{2}}}
$$

Tương tự như phương pháp góc nghiêng ngang, góc nghiêng của gradient ngang toàn phần sinh ra các giá trị cực đại trên ranh giới ngang của nguồn.

Cooper (2014) giới thiệu phương pháp góc nghiêng của biên độ tín hiệu giải tích. Trong phương pháp này, đạo hàm thẳng đứng của biên độ giải tích được chuẩn hóa bởi gradient ngang toàn phần của biên độ giải tích. Phương pháp được định nghĩa bởi biểu thức [20]:

$$
T A S=\operatorname{atan} \frac{\frac{\partial A S}{\partial z}}{\sqrt{\left(\frac{\partial A S}{\partial x}\right)^{2}+\left(\frac{\partial A S}{\partial y}\right)^{2}}}
$$

Theo Cooper (2014), sử dụng hàm TAS giúp giảm ảnh hưởng của vectơ từ hóa lên các kết quả xác định biên. Biên của vật thể được xác định bởi các đỉnh của hàm TAS [20].

Trong những năm gần đây, một số phương pháp cân bằng đã được phát triển để cải thiện độ phân giải của kết quả xác định biên. Dựa trên phương pháp bản đồ theta, Chen và nnk (2017) đã giới thiệu phương pháp bản đồ theta cải tiến. Phương pháp sử dụng các đạo hàm bậc cao hơn để tăng độ phân giải của các ranh giới ngang. Biểu thức toán học của phương pháp được cho bởi [21]:

$$
\mathrm{ITM}=\operatorname{acos} \frac{\sqrt{\left(\frac{\partial^{2} \mathrm{~F}}{\partial \mathrm{z} \partial \mathrm{x}}\right)^{2}+\left(\frac{\partial^{2} \mathrm{~F}}{\partial \mathrm{z} \partial \mathrm{y}}\right)^{2}}}{\left(\frac{\partial^{2} \mathrm{~F}}{\partial \mathrm{z} \partial \mathrm{x}}\right)^{2}+\left(\frac{\partial^{2} \mathrm{~F}}{\partial \mathrm{z} \partial \mathrm{y}}\right)^{2}+\left(\frac{\partial \mathrm{F}}{\partial \mathrm{z}} /(\mathrm{p} \times \mathrm{h})\right)^{2}}
$$

trong đó $\mathrm{h}$ là bước lấy mẫu và $\mathrm{p}$ là một tham số được quyết định bởi người phân tích. Tính toán thử nghiệm chỉ ra rằng giá trị của $\mathrm{p}$ trong khoảng từ 0.05 đến 5 sẽ sinh ra các kết quả tốt nhất (Chen và nnk, 2017). Tương tự như phương pháp bản đồ theta, phương pháp bản đồ theta cải tiến cung cấp các giá trị cực tiểu trên các ranh giới ngang nguồn. 
Một phương pháp cân bằng độ phân giải cao khác, được giới thiệu bởi Nasuti và nnk (2018) dựa trên gradient ngang toàn phần của hàm STDR. Phương pháp dựa trên tî̉ lệ giữa đạo hàm thẳng đứng bậc hai và gradient ngang toàn phần của hàm THG và được định nghĩa bởi biểu thức [22]:

$$
\mathrm{THG}_{\mathrm{STDR}}=\sqrt{\left(\frac{\partial \mathrm{STDR}}{\partial \mathrm{x}}\right)^{2}+\left(\frac{\partial \mathrm{STDR}}{\partial \mathrm{y}}\right)^{2}}
$$

trong đó hàm STDR được cho bởi biểu thức:

$$
\operatorname{STDR}=\operatorname{atan} \frac{M \times \frac{\partial^{2} \mathrm{~F}}{\partial \mathrm{z}^{2}}}{\sqrt{\left(\frac{\partial \mathrm{THG}}{\partial \mathrm{x}}\right)^{2}+\left(\frac{\partial \mathrm{THG}}{\partial \mathrm{y}}\right)^{2}}}
$$

với $\mathrm{M}$ là số thực dương và được quyết định bởi người phân tích. Về cơ bản, $\mathrm{M}$ có thể được định nghĩa là độ lớn của cường độ từ trường trung bình hoặc giá trị trọng lực tuyệt đối của khu vực [22].

\section{Tính toán thử nghiệm trên các mô hình}

Để đánh giá khả năng áp dụng của các phương pháp, trong phần này chúng tôi thiết kế một mô hình từ và một mô hình trọng lực cho việc tính toán thử nghiệm. Mỗi mô hình bao gồm ba nguồn lăng trụ được sắp xếp ở những độ sâu khác nhau. Điều này giúp khảo sát sự phụ thuộc của các phương pháp vào độ sâu của nguồn.

Hình 1a biểu diễn hình ảnh 3D của mô hình ba lăng trụ bị từ hóa. Hình $1 \mathrm{~b}$ biểu diễn hình chiếu của mô hình lên mặt phẳng nằm ngang. Các thông số hình học và vật lý của mô hình được đưa ra trong Bảng 1. Dựa trên các thông số này, dị thường từ gây bởi ba lăng trụ được tính toán trên mặt phẳng chứa $101 \times 201$ điểm quan sát với khoảng cách giữa các điểm là 1 km (Hình 1c).

a)

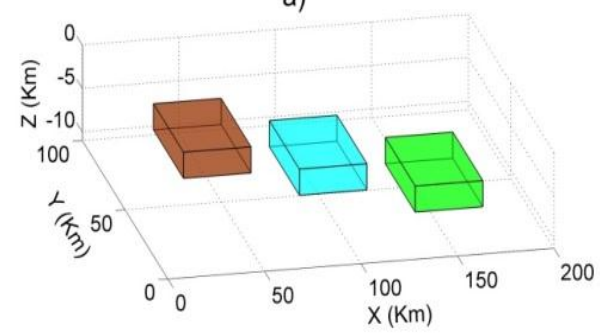

b)
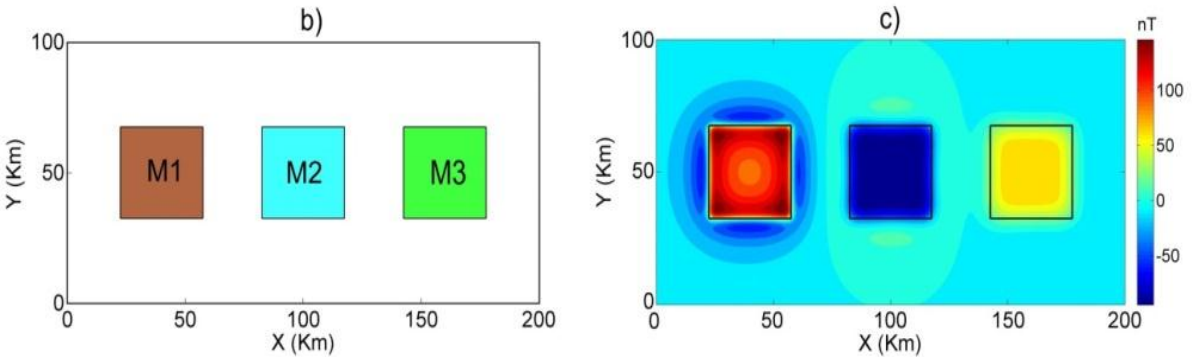

Hình 1: (a) Hình ảnh 3D của mô hình hóa, (b) Hình chiếu của mô hình lên mặt phẳng nằm ngang,

(c) Dị thường từ gây bởi mô hình. Các đường nét liền màu đen biểu diễn biên của vật thể.

Bảng 1. Các thông số hình học và từ hóa của mô hình từ

\begin{tabular}{|l|l|l|l|}
\hline Các thông số / ID & M1 & M2 & M3 \\
\hline Tọa độ tâm theo trục x $(\mathrm{km})$ & 40 & 100 & 160 \\
\hline Tọa độ tâm theo trục y $(\mathrm{km})$ & 50 & 50 & 50 \\
\hline Độ rộng $(\mathrm{km})$ & 35 & 35 & 35 \\
\hline
\end{tabular}




\begin{tabular}{|l|l|l|l|}
\hline Độ dài $(\mathrm{km})$ & 35 & 35 & 35 \\
\hline Độ sâu tới đỉnh $(\mathrm{km})$ & 2 & 5 & 8 \\
\hline Độ sâu tới đáy $(\mathrm{km})$ & 5 & 8 & 10 \\
\hline Độ từ khuynh $\left(^{\circ}\right)$ & 0 & 0 & 0 \\
\hline Độ từ thiên $\left(^{\circ}\right)$ & 90 & 90 & 90 \\
\hline Độ từ hóa $(\mathrm{A} / \mathrm{m})$ & 1 & -1 & 1 \\
\hline
\end{tabular}

Chúng tôi áp dụng các phương pháp đã trình bày ở trên cho dị thường từ trong Hình $1 \mathrm{c}$ và biểu diê̂n các kết quả thu được trên Hình 2. Có thể thấy từ Hình $2 \mathrm{a}$, phương pháp THG có thể xác định rõ ràng biên của nguồn nông $\mathrm{M} 1$ nhưng các tín hiệu trên biên của hai nguồn sâu hơn (M2 và M3) khá mờ nhạt. Tương tự như hàm THG, hàm AS cung cấp các kết quả rõ ràng cho nguồn nông M1 nhưng kém hiệu quả cho hai nguồn $\mathrm{M} 2$ và M3 (Hình 2b). Hơn nữa, khi nguồn nằm sâu (M3), các cực đại của hàm $\mathrm{AS}$ có xu hướng dịch chuyển vào trung tâm vật thể, khiến cho cấu trúc thu được trông nhỏ hơn cấu trúc thực (Hình $2 b$ ). Phương pháp TA đạt cực đại trên nguồn M1, M3 và đạt cực tiểu trên nguồn $\mathrm{M} 2$ (Hình $2 \mathrm{c}$ ). Mặc dù biên của cả ba vật thể được xác đinh bởi các đường đồng mức "không" trên bản đồ TA nhưng phương pháp sinh ra một số đường đồng mức "không" ảo trong bản đồ phân tích. Các đường đồng mức này không phản ánh bất kỳ ranh giới ngang nào. Mặc dù phương pháp THG_TA cung cấp các kết quả rõ ràng hơn so với phương pháp $\mathrm{TA}$, nhưng nó không thể cân bằng các dị thường có biên độ khác nhau và kết quả bị chi phối mạnh bởi dị thường gây bởi nguồn nông M1 (Hình 2d). Bên cạnh đó, phương pháp sinh ra các cạnh thứ cấp giữa các nguồn. Điều này, có thể dẫn đến những phân tích sai lệch khi xử lý các tài liệu thực tế. Hình $2 \mathrm{e}$ biểu diễn kết quả xác định biền theo phương pháp TM. Theo mô tả ban đầu của Wijns và cộng sự (2005), hàm TM cung cấp các giá trị cực đại trên biên của nguồn. Tuy nhiên, kết quả tính toán của chúng tôi chỉ ra rằng hàm TM sinh ra các giá trị cực tiểu trên biên của nguồn. Điều này cũng đã được chỉ ra trong các nghiên cứu được thực hiện bởi Ekinci và nnk (2013). Kết quả tính toán cũng chỉ ra rằng, mặc dù phương pháp $\mathrm{TM}$ có khả năng cân bằng các dị thường có biên độ khác nhau nhưng nó sinh ra các ranh giới sai lệch ở giữa các nguồn. Tương tự như phương pháp TM, hàm TDX có khả năng cân bằng các dị thường có biên độ khác sau nhưng nó sinh ra các ranh giới thứ cấp ở giữa các nguồn (Hình 2f). Phương pháp TTHG có thể xác định chính xác tất cả các biên của nguồn (Hình $2 \mathrm{~g}$ ). Do sử dụng tỉ lệ của các đạo hàm, phương pháp sinh ra các tín hiệu với cùng biên độ trên các ranh giới ngang của nguồn. Mặc dù không sinh ra bất cứ cạnh thứ cấp nào trong bản đồ phân tích, phương pháp TTHG có độ phân giải thấp, tức là các biên thu được có kích thước khá dày. Phương pháp TAS hiệu quả hơn hàm biên độ tín hiệu giải tích $\mathrm{AS}$ trong việc cân bằng các dị thường gây bởi các nguồn nằm ở những độ sâu khác nhau, nhưng với nguồn nằm sâu $\mathrm{M} 3$, các biên thu được có xu hướng trượt vào bên trong, khiến cho vật thể trông nhỏ hơn kích thước thực của nó (Hình $2 h$ ). Tương tự các phương pháp ở trên, sử dụng hàm TAS cũng cung cấp các kết quả với độ phân giải thấp. Hình $2 \mathrm{i}$ biểu diễn kết quả áp dụng phương pháp ITM với tham số $\mathrm{p}=2$. Có thể thây, phương pháp ITM có độ phân giải cao, các ranh giới thu được rất mảnh và xuất hiện tập trung trên biên của nguồn. Mặc dù có thể cân bằng các dị thường có biên độ khác nhau, phương pháp vẫn sinh ra các cạnh thứ cấp giữa các nguồn. Hình $2 \mathrm{j}$ biểu diễn kết quả áp dụng phương pháp THG STDR với $\mathrm{M}=50000$. So với các phương pháp kể trên, việc sử dụng phương pháp THG_STDR đem đến các kết quả với độ phân giải cao nhất. Theo Nasuti và nnk (2018), phương pháp có khả năng xác định nhiều cấu trúc chi tiết hơn so với các phương pháp khác [22]. Tuy nhiên, kết quả trên Hình $2 \mathrm{j}$ cho thấy, các cấu trúc chi tiết sinh ra bởi phương pháp THG_STDR là các cạnh thứ cấp xung quanh và ở trên các vật thể. Các cạnh này không phản ánh biên ngang của nguồn. Việc sinh ra nhiều cạnh thứ cấp có thể dẫn đến những phân tích sai khi áp dụng phương pháp cho các tài liệu thực tế. 

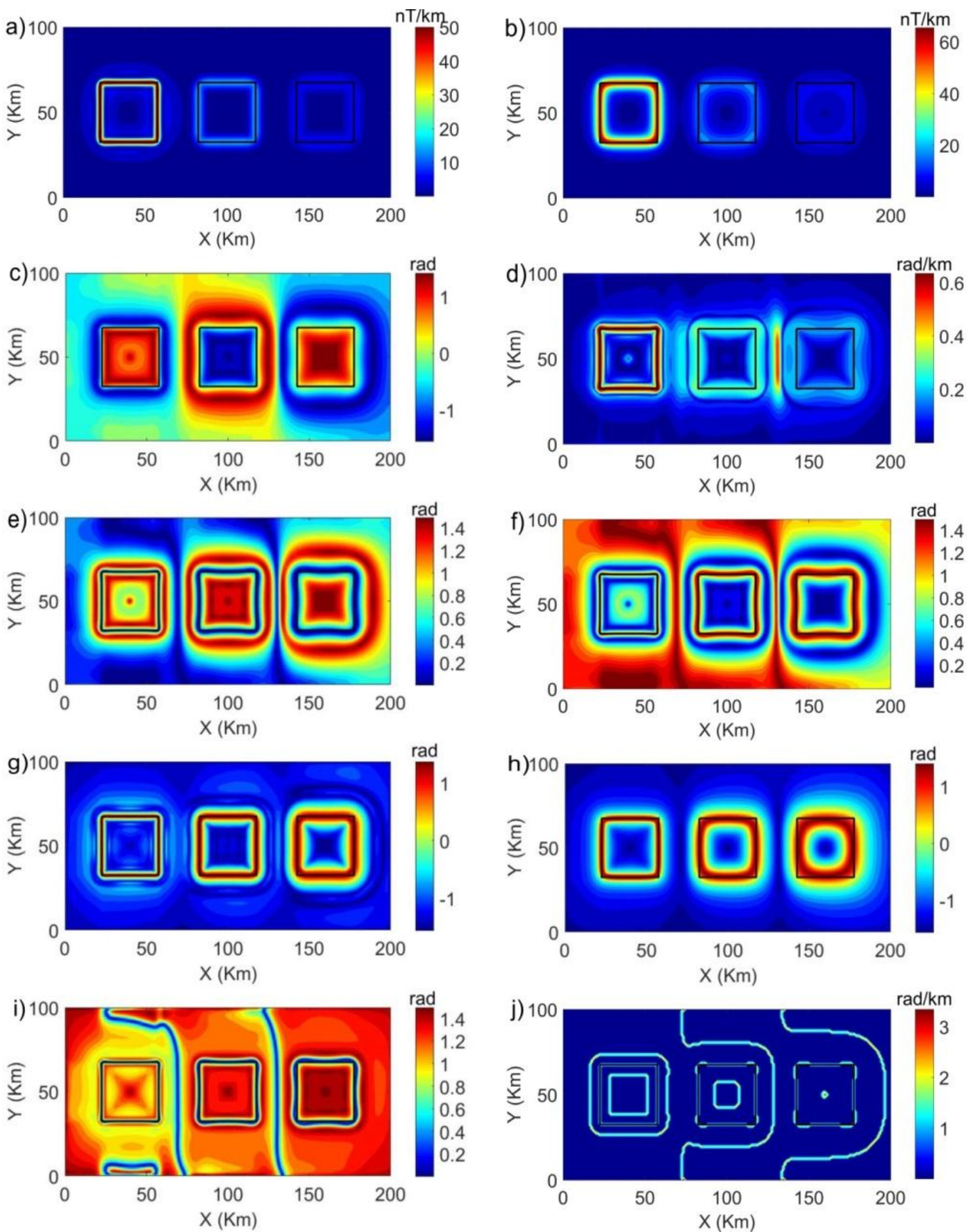

Hình 2. Kết quả xác định biên cho mô hình từ. (a) THG, (b) AS, (c) TA, (d) THG_TA, (e) TM, (f) TDX, (g) TTHG, (h) TAS, (i) ITM và (j) THG_STDR. Các đường nét liền màu đen biểu diễn biên ngang của các vật thể. 
Mô hình thứ hai được chúng tôi xem xét là mô hình trọng lực. Hình $3 \mathrm{a}$ biểu diễn hình ảnh $3 \mathrm{D}$ của mô hình và Hình $3 \mathrm{~b}$ biểu diễn hình chiếu của mô hình lên mặt phẳng nằm ngang. Các thông số hình học và mật độ của ba nguồn lăng trụ được liệt kê trong Bảng 2. Dựa trên các thông số này, dị thường trọng lực gây bởi ba lăng trụ được tính toán trên mặt phẳng chứa $101 \times 201$ điểm quan sát với khoảng cách giữa các điểm là $1 \mathrm{~km}$ (Hình $3 \mathrm{c}$ ). Khác với mô hình từ, trong mô hình trọng lực, chúng tôi còn xem xét đến hiệu quả của các phương pháp khi trường quan sát chứa nhiễu. Ở đây trường quan sát được cộng thêm nhiễu giả ngẫu nhiên với biên độ bằng $5 \%$ biên độ dị thường và được biểu diễn trên Hình
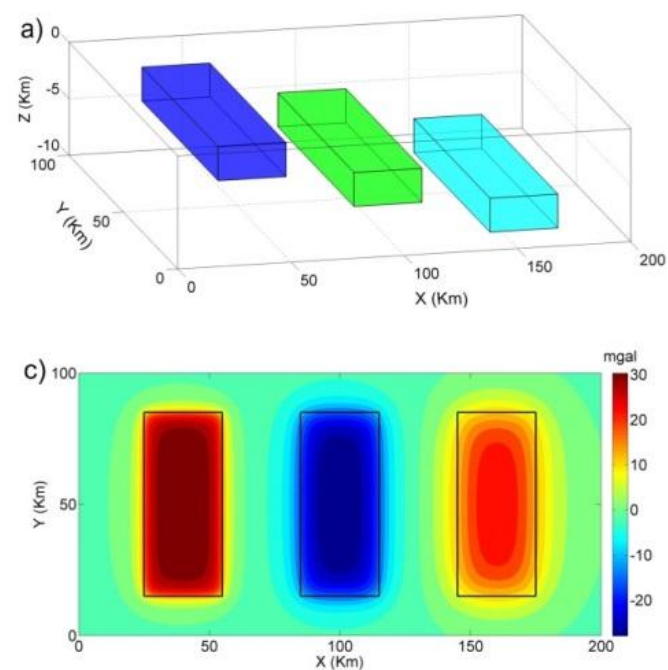

$3 d$. Vì các phương pháp xác định biên dựa trên việc tính toán các đạo hàm của dị thường trường thế nên các tín hiệu nhiễu trong bản đồ trường sẽ bị khuếch đại. Điều này có thể gây ảnh hưởng nghiêm trọng tới các kết quả phân tích. Để khắc phục hạn chế đó, thông thường người ta sử dụng các bộ lọc thông thấp loại bỏ các tín hiệu tần số cao gây bởi nhiễu. Một cách tiếp cận khác cũng khá phổ biến là sử dụng các bộ lọc nâng trường. Trong trường hợp này, chúng tôi thực hiện việc nâng trường lên độ cao $2 \mathrm{~km}$ trước khi áp dụng các phương pháp.
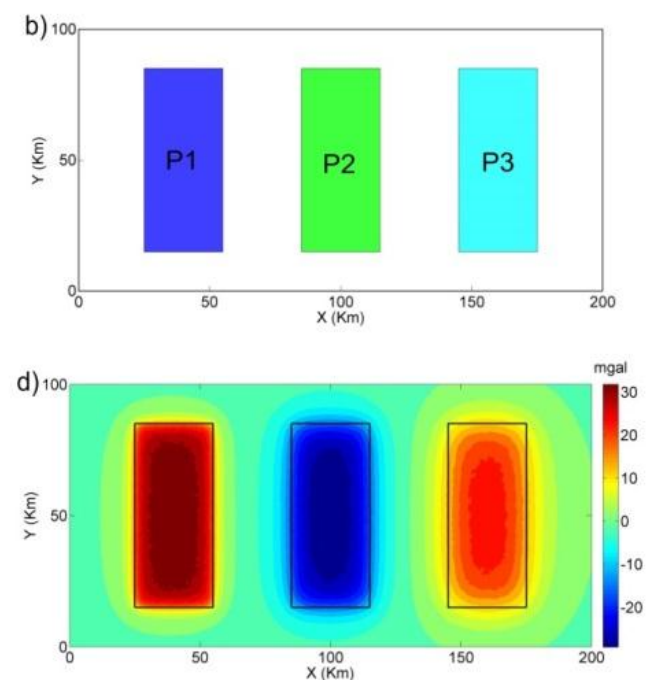

Hình 3. (a) Hình ảnh 3D của mô hình ba lăng trụ, (b) Hình chiếu của mô hình lên mặt phẳng nằm ngang, (c) Dị thường từ gây bởi mô hình, $(\mathrm{d})$ Dị thường từ được thêm nhiễu với biên độ bằng $5 \%$ biên độ dị thường. Các đường nét liền màu đen biểu diễn biên ngang của các vật thể.

Bảng 2. Các thông số hình học và từ hóa của mô hình trọng lực

\begin{tabular}{|l|l|l|l|}
\hline Các thông số / ID & P1 & P2 & P3 \\
\hline Tọa độ tâm theo trục x $(\mathrm{km})$ & 40 & 100 & 160 \\
\hline Tọa độ tâm theo trục y $(\mathrm{km})$ & 50 & 50 & 50 \\
\hline Độ rộng $(\mathrm{km})$ & 30 & 30 & 30 \\
\hline Độ dài $(\mathrm{km})$ & 70 & 70 & 70 \\
\hline Độ sâu tới đỉnh $(\mathrm{km})$ & 1 & 4 & 7 \\
\hline Độ sâu tới đáy $(\mathrm{km})$ & 4 & 7 & 10 \\
\hline Mật độ dư $\left(\mathrm{g} / \mathrm{cm}^{3}\right)$ & 0.3 & -0.3 & 0.3 \\
\hline
\end{tabular}



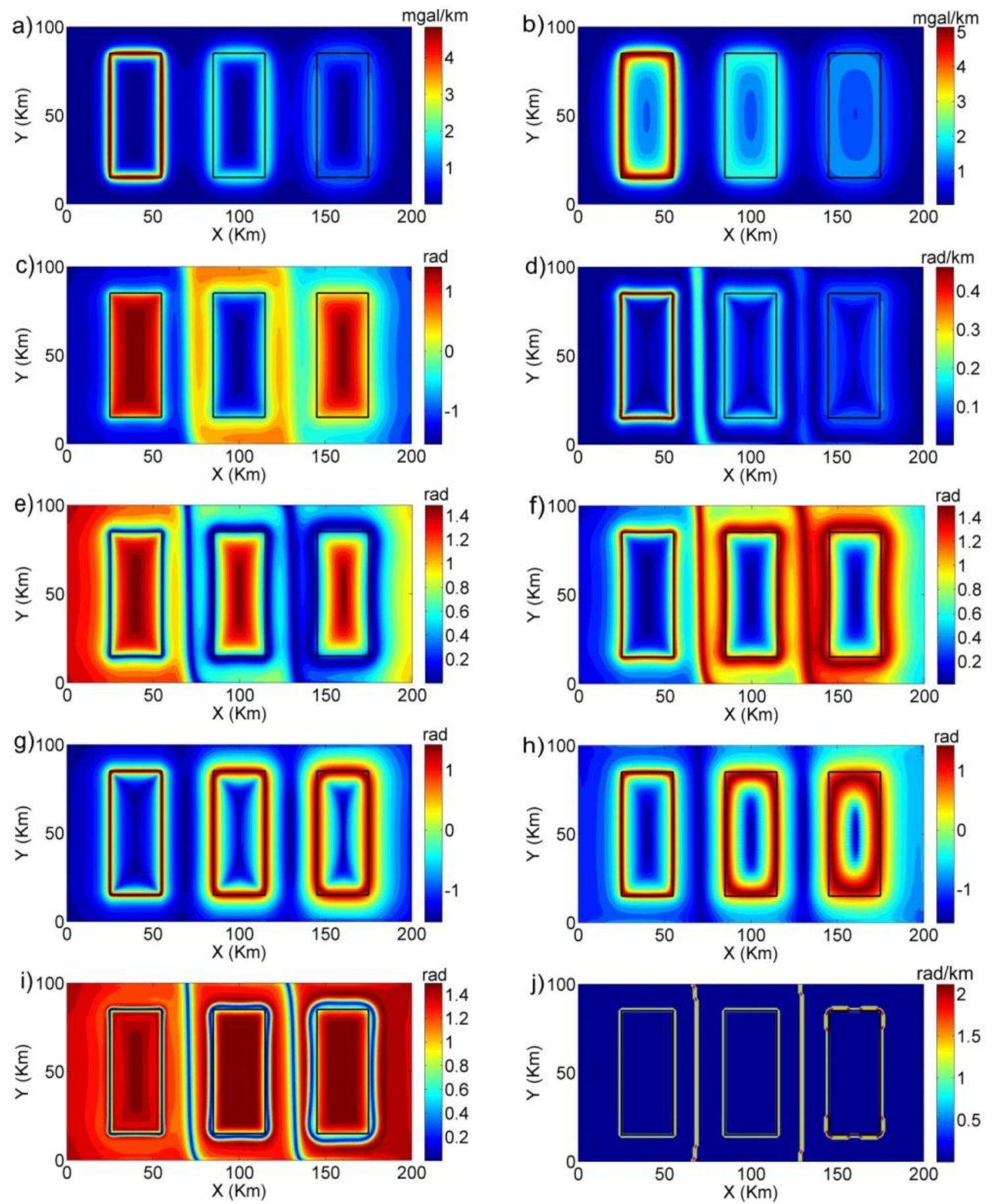

Hình 4. Kết quả xác định biên cho mô hình trọng lực không chứa nhiễu. (a) THG, (b) AS, (c) TA, (d) THG_TA, (e) TM, (f) TDX, (g) TTHG, (h) TAS, (i) ITM và (j) THG_STDR. Các đường nét liền màu đen biểu diễn biên. ngang của các vật thể. 
Hình 4 biểu diễn các kết quả xác định biên cho trường hợp trường trọng lực không chứa nhiễu. Có thể thấy, phương pháp THG bị chi phối mạnh bởi dị thường có biên độ lớn (Hình 4a). Phương pháp có thể xác định rõ ràng biên của nguồn nông $\mathrm{P} 1$ nhưng các tín hiệu trên biên của hai nguồn sâu hơn $(\mathrm{P} 2$ và $\mathrm{P} 3)$ tương đối mò̀ nhạt. Tương tự phương pháp THG, phương pháp AS chỉ hiệu quả trong việc xác định biên của nguồn nông $\mathrm{P} 1$, các biên thu được cho hai nguồn sâu hơn $\mathrm{P} 2$ và $\mathrm{P} 3$ không rõ ràng (Hình $4 \mathrm{~b})$. Hơn nữa, các biên của hai nguồn sâu bị trượt vào phía trong, khiến cho các vật thể xác định từ AS trông nhỏ hơn kích thước thực của chúng (Hình 4b). Hình $4 c$ biểu diễn kết quả áp dụng hàm TA. Có thể thấy, phương pháp TA sinh ra các giá trị cực đại trên các nguồn có mật độ dư dương ( $\mathrm{P} 1$ và $\mathrm{P} 3$ ) và đạt cực tiểu trên nguồn có mật độ dư âm (P2). Sử dụng phương pháp TA, biên của nguồn được xác định bởi các đường đồng mức "không". Mặc dù có thể xác định tất cả các ranh giới ngang của nguồn nhưng hàm $\mathrm{TA}$ sinh các cạnh thứ cấp giữa các nguồn. Hơn nữa, việc sử dụng các giá trị "không” để xác định biên có thể gặp khó khăn hơn khi đánh giá trực quan các kết quả so với các phương pháp xác định biên dựa trên giá trị cực đại hoặc cực tiểu. Mặc dù cung cấp các phản hồi sắc nét trên biên của nguồn $\mathrm{P} 1$ hơn phương pháp TA, nhưng phương pháp THG_TA không thề xác định rõ ràng biên ngang của hai nguồn sâu $\mathrm{P} 2, \mathrm{P} 3$ và vẫn sinh ra hai cạnh thứ cấp giữa các nguồn (Hình $4 \mathrm{~d}$ ). Các cực tiểu của $\mathrm{TM}$ nằm ngay trên biên của nguồn nông $\mathrm{P} 1$, tuy nhiên chúng bị trượt ra khỏi các biên của $\mathrm{P} 2$ và $\mathrm{P} 3$ (Hình $4 \mathrm{e}$ ). Kết quả tính toán chỉ ra rằng, vật thể nằm càng sâu, ranh giới thu được càng bị trượt ra xa khỏi biên thực và phương pháp $\mathrm{TM}$ vẫn sinh ra hai cạnh thứ cấp (Hình $4 \mathrm{e})$. Khác với hàm $\mathrm{TM}$, hàm TDX sử dụng các giá trị cực đại để xác định biên (Hình 4f). Phương pháp có thể xác định chính xác biên của nguồn nông $\mathrm{P} 1$ nhưng các cực đại của TDX bị trượt ra khỏi biên của $\mathrm{P} 2$ và $\mathrm{P} 3$. Phương pháp cũng sinh ra hai cạnh thứ thấp giữa các vật thể. Hình $4 \mathrm{~g}$ biểu diễn các biên thu được từ phương pháp TTHG. Có thể thấy từ hình vẽ, phương pháp TTHG không chỉ xác định chính xác biên ngang của các nguồn mà còn tránh sinh ra các cạnh thứ cấp trong bản đồ phân tích.
Phương pháp cung cấp các tín hiệu với cùng biên độ trên các cạnh của nguồn. Mặc dù hiệu quả hơn các phương pháp được sử dụng trước đó nhưng các cạnh thu được bởi phương pháp bị phân tán (có độ dày rộng). Hình $4 \mathrm{~h}$ biểu diễn kêt quả áp dụng phương pháp TAS. Phương pháp xác định chính xác biên của nguồn nông $\mathrm{P} 1$ nhưng kém chính xác hơn với $\mathrm{P} 2$ và $\mathrm{P} 3$. Sử dụng phương pháp TAS có thể cân bằng các dị thường có biên độ khác nhau nhưng các cực đại của TAS bị trượt vào trong vật thể khi nó nằm sâu. Hình $4 \mathrm{i}$ biểu diễn kết quả áp dụng phương pháp ITM. Chúng ta có thể thấy rằng, phương pháp bản đồ theta cải tiến ITM cung cấp các ranh giới ngang với độ phân giải rất cao, các ranh giới thu được rất mảnh và rõ ràng. Tuy nhiên, phương pháp không khắc phục được những nhược điểm của phương pháp bản đồ theta ban đầu. Tức là, vẫn sinh ra các cạnh thứ cấp trong bản đồ phân tích và với nguồn nằm sâu, các ranh giới thu được bị trượt ra khỏi cấu trúc thực. Hình $4 \mathrm{j}$ biểu diễn kết quả áp dụng phương pháp THG_STDR với $\mathrm{M}=90.000$. Tương tự phương pháp ITM, phương pháp THG_STDR có thể cung cấp các kết quả với độ phân giải rất cao. So với ITM, hàm THG_STDR có thể xác định các cạnh của nguồn sâu chính xác hơn với các cực đại nằm ngay trên biên của nguồn. Mặc dù hiệu quả hơn ITM, phương pháp vẫn không thể tránh được việc sinh ra hai biên ảo giữa các vật thể.

Hình 5 biểu diễn các kết quả xác định biên cho trường hợp trường trọng lực chứa nhiễu. Hình $5 \mathrm{a}$ và $5 \mathrm{~b}$ lần lượt biểu diễn kết quả áp dụng phương pháp THG và $\mathrm{AS}$. Có thể thấy phương pháp THG hiệu quả hơn AS trong trường hợp các nguồn nằm sâu. Tuy nhiên cả hai phương pháp đều không thể cân bằng các dị thường có biên độ khác nhau. Hơn nữa kết quả thu được từ hai phương pháp đều có độ phân giải thấp. Hình $5 \mathrm{c}$ biểu diễn kết quả áp dụng phương pháp TA. Quan sát trên hình vẽ có thể thấy, hàm TA không cung cấp các phản hồi sắc nét trên biên của vật thể. Các đường đồng mức "không" trên bản đồ TA bị trượt ra ngoài biên thực trong trường hợp các nguồn nằm sâu. Bên cạnh đó, phương pháp sinh ra các đường đồng mức "không" thứ cấp giữa các nguồn. Hình $5 \mathrm{~d}$ biểu diễn kết quả áp dụng phương pháp THG_TA. Do sử dụng các 
đạo hàm của TA, phương pháp THG_TA bị ảnh hưởng mạnh bởi nhiễu. Phương pháp không thể cung cấp các tín hiệu cân bằng và sinh ra hai ranh giới ảo giữa các nguồn. Hình $5 \mathrm{e}$ và $5 \mathrm{f}$ lần lượt biểu diễn các ranh giới thu được theo phương pháp TM và TDX. Cả hai phương pháp cho các kết quả tương tự. Không chỉ kém hiệu quả trong việc đánh giá các ranh giới ngang của nguồn sâu, cả hai phương pháp đều sinh ra các ranh giới ảo giữa các nguồn. Hình $5 \mathrm{~g}$ và $5 \mathrm{~h}$ lần lượt biểu diễn các ranh giới thu được theo phương pháp TTHG và $\mathrm{TAS}$. Trong khi hàm TTHG đạt cực đại trên biên của tất cả các vật thể, các cực đại thu được bởi hàm TAS bị trượt vào trong vật thể khi các
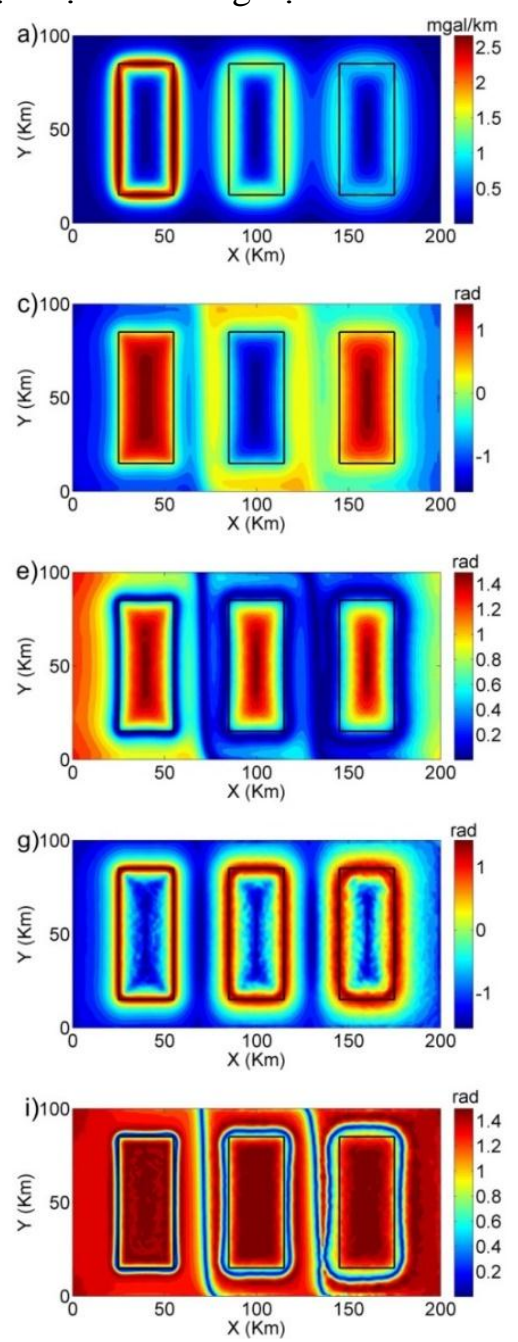

nguồn nằm sâu. Do sử dụng các đạo hàm bậc cao của đạo hàm thẳng đứng, phương pháp TAS bị ảnh hưởng mạnh bởi nhiễu. Hình $5 i$ và $5 \mathrm{j}$ lần lượt biểu diễn các ranh giới thu được theo phương pháp ITM và THG_STDR. Quan sát trên các hình vẽ có thể nhận thấy, trong trường hợp này, cả ITM và THG STDR đều cung cấp các hình ảnh với độ phân giải rất cao. Các ranh giới thu được xuất hiện theo cách rất tập trung. Mặc dù phương pháp THG_STDR chính xác hơn phương pháp ITM nhưng cũng không thể tránh được việc sinh ra các cạnh thứ cấp trong các bản đồ phân tích.
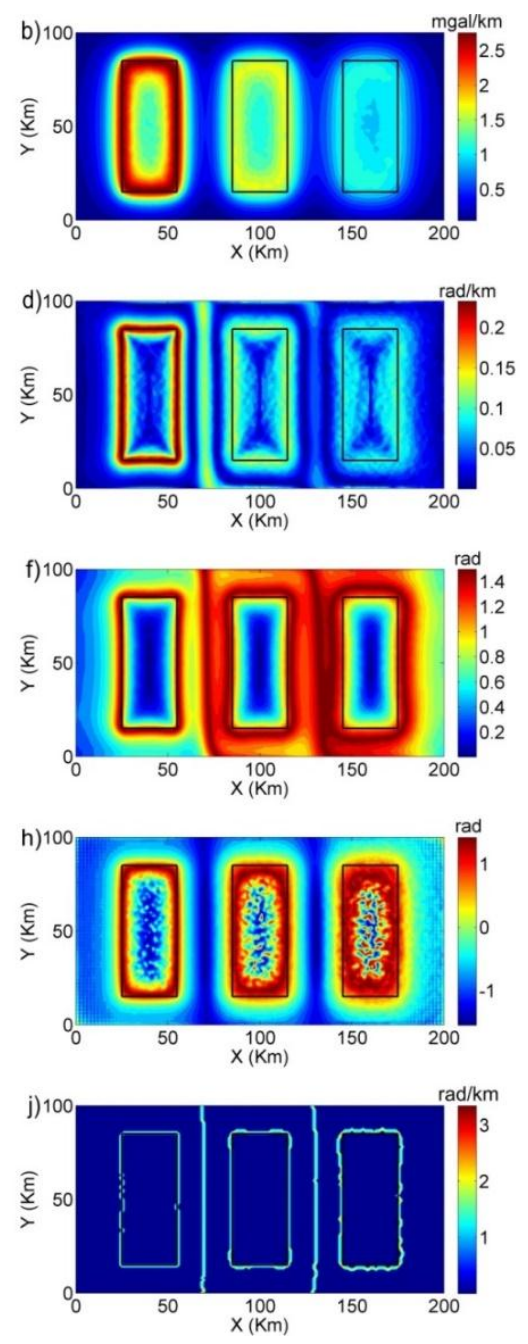

Hình 5. Kết quả xác định biên cho mô hình trọng lực chứa nhiễu. (a) THG, (b) AS, (c) TA,

(d) THG_TA, (e) TM, (f) TDX, (g) TTHG, (h) TAS, (i) ITM và (j) THG_STDR.

Các đường nét liền màu đen biểu diễn biên ngang của các vật thể. 


\section{Kết quả áp dụng thực tế}

Khả năng áp dụng thực tế của các phương pháp THG, AS, TA, THG_TA, TM, TDX, TTHG, TAS, ITM và THG_STDR được đánh giá thông qua việc phân tích bản đồ dị thường từ của khu vực Zhurihe (Trung Quốc) và bản đồ trọng lực Bouguer từ một khu vực thuộc miền Bắc Việt Nam.

\subsection{Kết quả phân tích bản đồ dị thuờng tù̀ khu vục Zhurihe, Trung Quốc}

Trước tiên, chúng tôi xem xét hiệu quả áp dụng thực tế của các phương pháp thông qua việc phân tích bản đồ dị thường từ khu vực Zhurihe. Khu vực nghiên cứu nằm ở phía Đông Bắc Trung Quốc với kích thước $8468 \mathrm{~km}^{2}$. Hình $6 \mathrm{a}$ biểu diễn bản đồ địa chất của khu vực Zhurihe (Ma và nnk, 2014). Khu vực Zhurihe bị bao phủ hầu hết bởi các trầm tích lục địa, ngoại trừ một số đai cơ (dyke) sa thạch giàu sắt có hướng Tây Bắc Đông Nam [29]. Hình $6 \mathrm{~b}$ biểu diễn bản đồ dị thường từ được tính chuyển về cực của khu vực trên mặt phẳng chứa $74 \times 117$ điểm quan sát với khoảng cách lưới là $1 \mathrm{~km}$ [19]. Hình $6 \mathrm{c}$ biểu diễn kết quả áp dụng phương pháp THG lên dị thường từ chuyển về cực. Có thể thấy, phương pháp THG bị chi phối mạnh bởi các dị thường biên độ lớn, do đó các kết quả thu được trên bản đồ THG khá mờ nhạt. Hình $6 \mathrm{~d}$ biểu diễn kết quả áp dụng phương pháp AS lên dị thường từ chuyển về cực. Tương tự phương pháp THG, phương pháp AS bị chi phối mạnh bởi các dị thường biên độ lớn, do đó không thể xác định được các cấu trúc gây dị thường yếu. Kết quả trên bản đồ AS không phân định rõ ràng các thành tạo giàu sắt và đai cơ. Hình 6e trình bày kết quả áp dụng phương pháp TA. Mặc dù rất hiệu quả trong việc cân bằng đồng thời các dị thường có biên độ lớn và nhỏ, phương pháp TA không thể cung cấp các phản hồi rõ ràng trên các ranh giới địa chất. Hình 6f trình bày kết quả áp dụng phương pháp THG_TA. Có thể thấy, phương pháp THG TA cung cấp các kết quả khá rời rạc và không thể cân bằng các dị thường có biên độ khác nhau. Hình $6 \mathrm{~g}$ trình bày các ranh giới thu được từ phương pháp TM. Quan sát từ hình vẽ, có thể nhận thấy, một số đường đồng mức âm trong bản đồ TM chạy dọc theo ranh giới của các cấu trúc granit. Tuy nhiên, các ranh giới trong bản đồ TM bị kết nối lại với nhau. Điều này vô tình gây khó khăn trong phân định các cấu trúc địa chất riêng biệt. Hình 6 h trình bày kết quả áp dụng phương pháp TDX. Có thể thấy, một số cực đại của TDX có mối tương quan tốt với các ranh giới granit. Tuy nhiên, tương tự phương pháp TM, các cực đại của TDX bị kết nối lại với nhau, gây khó khăn trong việc phân định các cấu trúc thực. Hình 6i biểu diễn các ranh giới địa chất được xác định theo phương pháp TTHG. Có thể thấy các cực đại của TTHG nằm trên hầu hết các ranh giới granit. Các cực đại đó cũng phản ánh tốt các cấu trúc hướng Tây Bắc - Đông Nam trong bản đồ địa chất. Hình $6 \mathrm{j}$ biểu diê̂n kết quả áp dụng phương pháp TAS. Mặc dù có thể cân bằng các dị thường có biên độ khác nhau, phương pháp không xác định rõ ràng các cấu trúc có hướng Tây Bắc - Đông Nam. Hình $6 \mathrm{k}$ và 61 lần lượt biểu diễn kết quả áp dụng các phương pháp ITM và THG_STDR. So với các phương pháp khác, hai phương pháp ITM và THG_STDR cung cấp các kết quả với độ phân giải căo hơn rất nhiều. Các ranh giới từ tính thu được trên bản đồ ITM và THG_STDR rất sắc nét. Tuy nhiên, như đã thảo luận trong các mô hình, việc sử dụng hai phương pháp kể trên có thể dẫn đến việc sinh ra các ranh giới thứ cấp. Bên cạnh đó, các ranh giới thu được trong bản đồ ITM và THG STDR cũng bị kết nối lại với nhau. Điều này có thể dẫn đến những phân tích sai lệch về cấu trúc địa chất khu vực.

\subsection{Kết quả phân tích bản đồ dị thường trọng lục Bouguer miền Bắc Việt Nam}

Trong ví dụ thực tế thứ hai, chúng tôi tiếp tục áp dụng các phương pháp để phân tích bản đồ dị thường trọng lực Bouguer của một khu vực thuộc phía Bắc Việt Nam. Bản đồ địa chất khu vực được biểu diễn trên Hình $7 \mathrm{a}$ [30]. Khu vực nghiên cứu bao gồm năm đơn vị cấu trúc địa chất chính, từ Đông Bắc đến Tây Nam gồm: bồn trũng An Châu, bồn trũng Hà Nội, khối kết tinh Fan Si Pan, phức hệ Sông Đà và nếp lồi Sông Mã [31]. Các đứt gãy Sông Hồng và Sông Chảy là các đứt gãy chính của khu vực. 


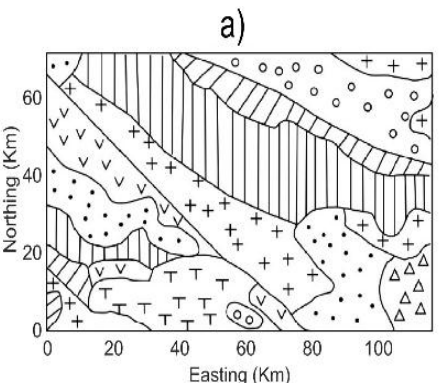

b)

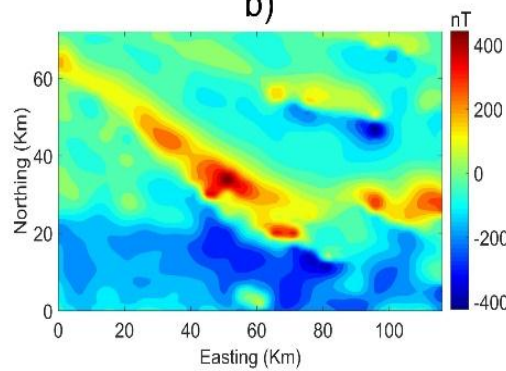

e)

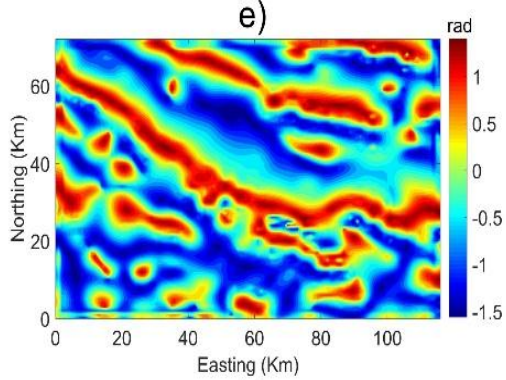

h)

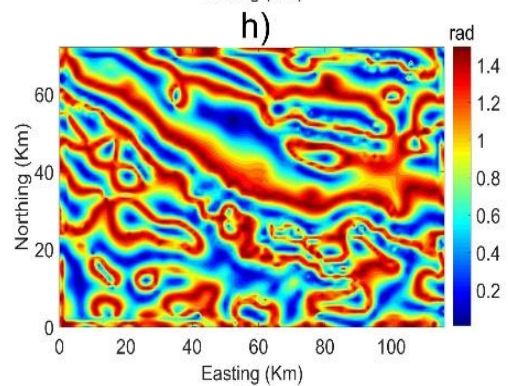

k)

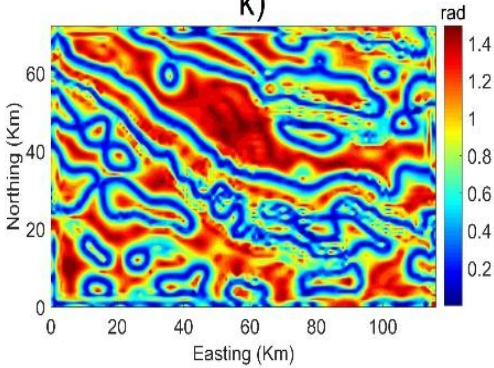

$\checkmark$ Silurian

$++_{+}^{+}$Granite

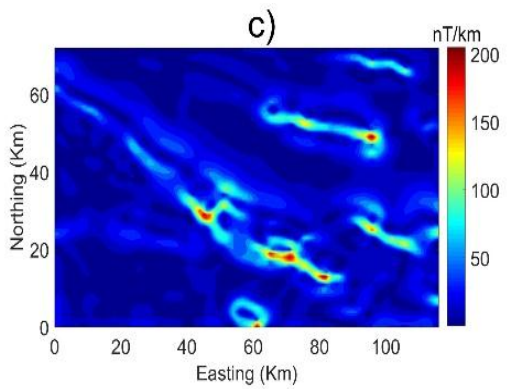

f)

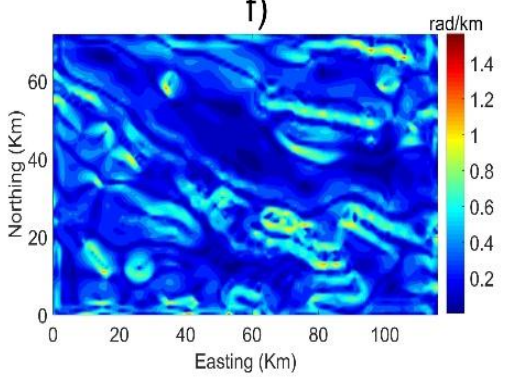

i)

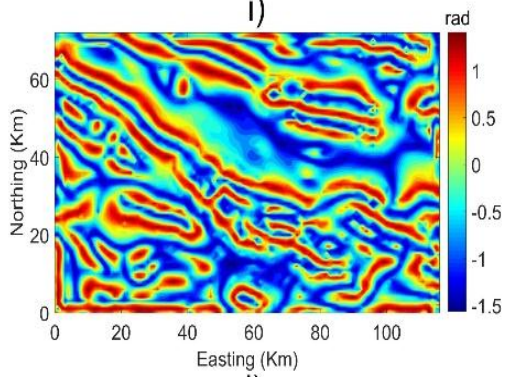

l)

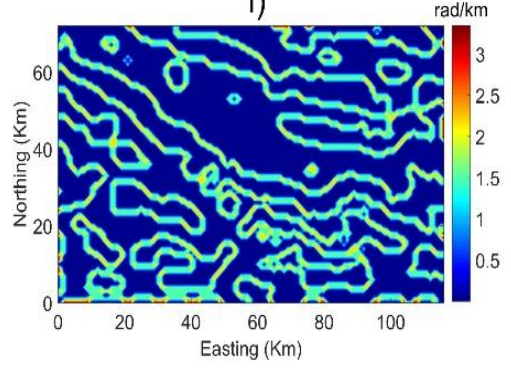

$\Delta \Delta$ Diorite

$T_{T}$ Triassic

Hình 6. (a) Bản đồ địa chất khu vực Zhurihe, Trung Quốc, (b) Bản đồ dị thường từ chuyển về cực của khu vực Zhurihe, (c) THG, (d) AS, (e) TA, (f) THG_TA, (g) TM, (h) TDX, (i) TTHG, (j) TAS, (k) ITM và (l) THG_STDR. 

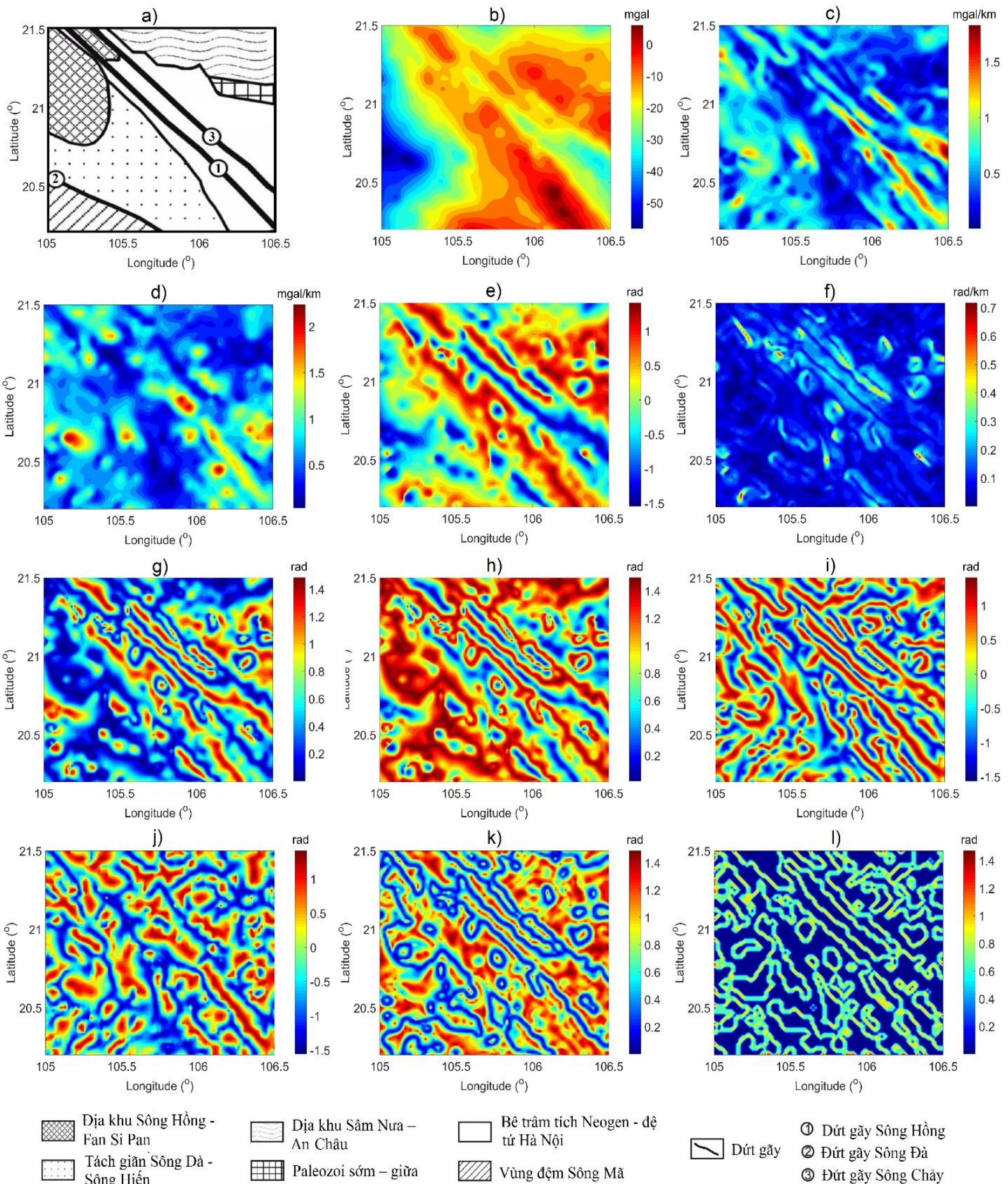

Dịa khu Sâm Nưa -

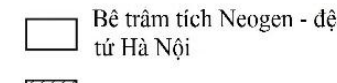

Paleozoi sớm - giữa

Vủng đệm Sông Mã

Hình 7. (a) Bản đồ địa chất khu vực nghiên cứu, (b) Bản đồ dị thường trọng lực Bouguer khu vực trũng Sông Hồng, (c) THG, (d) AS, (e) TA, (f) THG_TA, (g) TM, (h) TDX, (i) TTHG, (j) TAS, (k) ITM và (1) THG_STDR. 
Hình $7 \mathrm{~b}$ biểu diễn bản đồ trọng lực Bouguer của khu vực. Bản đồ này được thành lập bởi Tổng cục Địa chất và Khoáng sản Việt Nam [7]. Để giảm ảnh hưởng của nhiê̂u, trường trọng lực được tính chuyển trường lên độ cao $1 \mathrm{~km}$ trước khi áp dụng các phương pháp. Hình $7 \mathrm{c}$ và $7 \mathrm{~d}$ lần lượt biểu diễn các ranh giới thu được từ phương pháp THG và $\mathrm{AS}$. Cả hai phương pháp đều không thể cân bằng các dị thường có biên độ khác nhau. Quan sát hai bản đồ có thể nhận thấy, phương pháp THG có thể xác định hai đứt gãy Sông Hồng và Sông Chảy tốt hơn phương pháp AS. Tuy nhiên các kết quả thu được tương đối mờ nhạt. Hình $7 \mathrm{e}$ và $7 \mathrm{f}$ lần lượt biểu diễn kết quả xác định biên theo hàm $\mathrm{TA}$ và gradient ngang toàn phần của nó (THG TA). Phương pháp TA có thể cân bằng các dị thường có biên độ khác nhau nhưng không cung cấp các phản hồi sắc nét trên các ranh giới địa chất. Trong trường hợp này, phương pháp THG TA rất kém hiệu quả. Các tín hiệu trên bản đồ THG_TA rất mờ nhạt và xuất hiện một cách rời rạc. Hình $7 \mathrm{~g}$ và $7 \mathrm{~h}$ lần lượt biểu diễn kết quả xác định biên theo phương pháp TM và $T D X$. Mặc dù các ranh giới thu được từ hai phương pháp phản ánh các cấu trúc hướng Tây Bắc - Đông Nam của khu vực, nhưng các ranh giới đó bị kết nối lại với nhau. Điều này có thể dẫn đến những nhận định không chính xác về các ranh giới địa chất của khu vực. Hình $7 \mathrm{i}$ biểu diễn các cấu trúc thu được từ phương pháp TTHG. Có thể thấy, các cực đại của TTHG không chỉ khẳng định sự tồn tại của đứt gãy Sông Hồng và Sông Chảy mà còn chỉ ra một loạt các cấu trúc không xuất hiện trên bản đồ địa chất. Mặc dù phương pháp này cung cấp các ranh giới rõ ràng hơn các phương pháp được sử dụng trước đó nhưng các kết quả thu được có độ phân giải thấp. Hình $7 \mathrm{j}$ biểu diễn bản đồ TAS. Nhìn vào bản đồ, chúng ta có thể nhận thấy, phương pháp TAS cung cấp các kết quả khá tương tự phương pháp TTHG. Tuy nhiên, các kết quả thu được bị phân tán và thiếu sự liên tục. Phương pháp dường như cũng kém hiệu quả trong việc xác định các cấu trúc gần nhau. Hình $7 \mathrm{k}$ và 71 lần lượt biểu diễn kết quả xác định biên theo phương pháp ITM và THG_STDR. Mặc dù khắc phục được hạn chế về độ phân giải của các phương pháp kể trên, các tín hiệu trong bản đồ ITM và THG_STDR bị kết nối lại với nhau. Hơn nữa, trong trường hợp này, trường quan sát chứa đồng thời các dị thường dương và âm, nên cả hai phương pháp ITM và THG STDR đều sinh các cấu trúc ảo trong các bản đồ phân tích. Do đó, việc sử dụng hai phương pháp độ phân giải cao có thể dẫn đến những kết luận sai lầm về cấu trúc địa chất của khu vực.

\section{Kết luận}

Trong nghiên cứu này, chúng tôi đã đánh giá hiệu quả của các phương pháp xác định biên được sử dụng phổ biến và một số phương pháp được phát triển gần đây. Từ những kết quả thu được trên các mô hình lý thuyết và tài liệu thực tế, chúng tôi có một số kết luận sau:

Ba phương pháp THG, AS và THG_TA không thể cân bằng các dị thường có biên độ khác nhau, do đó kém hiệu quả trong việc xác định biên của các cấu trúc nằm sâu. Bên canh đó, phương pháp THG_TA có thể sinh ra các cạnh thứ cấp trong các bản đồ phân tích.

Phương pháp $T A, T M$ và $T D X$ có thể cân bằng đồng thời các dị thường có biên độ lớn và nhỏ, nhưng lại sinh ra các biên thứ cấp khi mô hình chứa đồng thời các nguồn có độ từ hóa dương và âm hoặc chứa đồng thời các nguồn có mật độ dư dương và âm. Hơn nữa, các kết quả thu được từ các phương pháp có thể lớn hơn cấu trúc thực khi các nguồn nằm sâu.

Phương pháp TAS có thể xác định rõ ràng ranh giới của nguồn nông. Khi các nguồn nằm sâu, các cực đại của TAS bị trượt vào phía trong vật thể, khiến các cấu trúc thu được nhỏ hơn cấu trúc thực.

Hai phương pháp ITM và THG_STDR cung cấp các kết quả với độ phân giải rất cao, tuy nhiên chúng sinh ra nhiều cấu trúc ảo trong bản đồ phân tích. Bên cạnh đó, các kết quả thu được từ hai phương pháp bị kết nối lại với nhau, gây khó khăn cho việc phân tích.

Phương pháp TTHG có thể xác định tất cả các biên ngang của nguồn mà không sinh ra bất 
kỳ biên ảo nào. Kết quả thu được từ phương pháp trùng khớp với mô hình lý thuyết và các ranh giới từ tính/mật độ thu được trên các tài liệu thực tế cũng phù hợp với cấu trúc địa chất đã biết trong các khu vực. Tuy nhiên, các kết quả thu được từ phương pháp TTHG có độ phân giải thấp hơn phương pháp ITM và THG_STDR.

\section{Lời cảm ơn}

Nghiên cứu sinh được tài trợ bởi Tập đoàn Vingroup - Công ty $\mathrm{CP}$ và hỗ trợ bởi Chương trình học bổng đào tạo thạc sĩ, tiến sĩ trong nước của Quỹ Đổi mới sáng tạo Vingroup (VINIF), Viện Nghiên cứu Dữ liệu lớn (VINBIGDATA)".

\section{Tài liệu tham khảo}

[1] S.K. Hsu, D. Coppense, C.T. Shyu, Highresolution detection of geologic boundaries from potential field anomalies: An enhanced analytic signal technique, Geophysics 61(1996) 1947 1957. https://doi.org/10.1190/1.1443966.

[2] M. Fedi, G. Florio, Detection of potential fields source boundaries by enhanced horizontal derivative method, Geophysical Prospecting 49(2001) 40-58. https://doi.org/10.1046/j.13652478.2001.00235.x.

[3] F.J.F. Ferreira, J. de Souza, A.B.E.S de Bongiolo, L.G. de Castro, Enhancement of the total horizontal gradient of magnetic anomalies using the tilt angle, Geophysics 78(2013) J33-J41. https://doi.org/10.1190/geo2011-0441.1.

[4] A.M. Eldosouky, L.T. Pham, H. Mohammed, B. Pradhan, A comparative study of THG, AS, TA, Theta, TDX and LTHG techniques for improving source boundaries detection of magnetic data using synthetic models: a case study from G. Um Monqul, North Eastern Desert, Egypt, Journal of African Earth Sciences 170(2020) 103940. https://doi.org/10.1016/j.jafrearsci.2020.103940.

[5] E. Oksum, M.N. Dolmaz, L.T. Pham, Inverting gravity anomalies over the Burdur sedimentary basin, SW Turkey, Acta Geodaetica et Geophysica 54(2019) 445-460. https://doi.org/10.1007/s40328 019-00273-5.

[6] A.M. Eldosouky, Aeromagnetic data for mapping geologic contacts at Samr El-Qaa area, North Eastern Desert, Egypt, Arab J Geosci. 12(2019) 2. https://doi.org/10.1007/s12517-018-4182-2.
[7] L.T. Pham, A comparative study on different filters for enhancing potential field source boundaries: synthetic examples and a case study from the Song Hong Trough (Vietnam), Arabian Journal of Geosciences 13(2020) 723. https://doi. org/10.1007/s12517-020-05737-5.

[8] L.T. Pham, M. Le-Huy, E. Oksum, T.D. Do, Determination of maximum tilt angle from analytic signal amplitude of magnetic data by the curvature-based method, Vietnam Journal of Earth Sciences 40(2018) 354-366. https://doi.org/10. 15625/0866-7187/40/4/13106

[9] L.T. Pham, E. Oksum, T.D. Do, M.D. Vu, Comparison of different approaches of computing the tilt angle of the total horizontal gradient and tilt angle of the analytic signal amplitude for detecting source edges, Bulletin of the Mineral Research and Exploration 16(2020). https://doi.org/10. 19111/bulletinofmre.746858.

[10] H.M. Evjen, The place of the vertical gradient in gravitational interpretations, Geophysics 1(1936) 127-136. https://doi.org/10.1190/1.1437067.

[11] L. Cordell, V.J.S. Grauch, Mapping basement magnetization zones from aeromagnetic data in the San Juan basin, New Mexico. In: Hinze WJ (ed) The utility of regional gravity and magnetic anomaly maps. Society of Exploration Geophysics, Tulsa, (1985) 181-197. https://doi. org/10.1190/1.0931830346.

[12] W.R.J Roest, J. Verhoef, M. Pilkington, Magnetic interpretation using the 3-D analytic signal, Geophysics 57(1992) 116-125. https://doi.org/ $10.1190 / 1.1443174$

[13] B. Verduzco, J.D. Fairhead, C.M. Green, C. MacKenzie, New insights into magnetic derivatives for structural mapping, The Leading Edge 23(2004) 116-119. https://doi.org/10.1190/ 1.1651454

[14] F. Cella, M. Fedi, G. Florio, Toward a full multiscale approach to interpret potential fields, Geophys. Prospect. 57 (2009) 543-557. https:// doi.org/10.1111/j.1365-2478.2009.00808.x.

[15] M. Beiki, Analytic signals of gravity gradient tensor and their application to estimate source location, Geophysics 75 (2010) 159-174. https:// doi.org/10.1190/1.3493639.

[16] H.G. Miller, V. Singh, Potential field tilt a new concept for location of potential field sources, Journal of Applied Geophysics 32(1994) 213-217. https://doi.org/10.1016/0926-9851(94) 90022-1.

[17] C. Wijns, C. Perez, P. Kowalczyk, Theta map: Edge detection in magnetic data, Geophysics 70(2005) 39-43. https://doi.org/10.1190/1.1988184.

[18] G.R.J. Cooper, D.R. Cowan, Enhancing potential field data using filters based on the local phase, 
Computers \& Geosciences 32(2006) 1585-1591. https://doi.org/10.1016/j.cageo.2006.02.016.

[19] Y. Yuan, Q. Yu, Edge detection in potential-field gradient tensor data by use of improved horizontal analytical signal methods, Pure and Applied Geophysics 172(2014) 461-472. https://doi.org/ 10.1007/s00024-014-0880-1.

[20] G.R.J. Cooper, Reducing the dependence of the analytic signal amplitude of aeromagnetic data on the source vector direction, Geophysics 79(2014) J55-J60. https://doi.org/10.1190/geo2013-0319.1.

[21] A.G. Chen, T.F. Zhou, D.J. Liu, S. Zhang, Application of an enhanced theta-based filter for potential field edge detection: a case study of the Luzong ore district, Chin. J. Geophys. 60(2017) 203-218. https://doi.org/10.1002/cjg2.30039.

[22] Y. Nasuti, A. Nasuti, D. Moghadas, STDR: A novel approach for enhancing and edge detection of potential field data, Pure and Applied Geophysics 176(2018) 827-841. https://doi.org/ 10.1007/s00024-018-2016-5.

[23] L.T. Pham, E. Oksum, T.D. Do, M. Le-Huy, New method for edges detection of magnetic sources using logistic function, Geofizichesky Zhurnal 40(2018) 127-135. https://doi.org/10.24028/ gzh.0203-3100.v40i6.2018.151033.

[24] L.T. Pham, T.D. Do, E. Oksum, A new method for edge detection in interpretation of potential field data, Journal of Engineering Sciences and Design 6(2018) 637-642. https://doi.org/10.21923/jesd. 441090.

[25] L.T. Pham, E. Oksum, T.D. Do, Edge enhancement of potential field data using the logistic function and the total horizontal gradient, Acta Geodaetica et Geophysica 54(2019) 143155. https://doi.org/10.1007/s40328-019-00248-6.
[26] L.T. Pham, E. Oksum, T.D. Do, M. Le-Huy, M.D. $\mathrm{Vu}$, V.D. Nguyen, LAS: A combination of the analytic signal amplitude and the generalised logistic function as a novel edge enhancement of magnetic data, Contributions to Geophysics and Geodesy 49(2019) 425-440. https://doi.org/10. 2478/congeo-2019-0022.

[27] L.T. Pham, T.V. Vu, S. Le-Thi, P.T. Trinh, Enhancement of potential field source boundaries using an improved logistic filter, Pure Appl. Geophys. (2020) https://doi.org/10.1007/ s00024-020-02542-9.

[28] L.T. Pham, A.M. Eldosouky, E. Oksum, S.A. Saada, A new high resolution filter for source edge detection of potential data, Geocarto International. (2020) doi.org/10.1080/10106049.2020.1849414.

[29] G. Ma, C. Liu, L. Li, Balanced horizontal derivative of potential field data to recognize the edges and estimate location parameters of the source, Journal of Applied Geophysics 108(2014) 12-18. https://doi.org/10.1016/j.jappgeo.2014.06. 005.

[30] P.T. Hieu, F. Chen, L.T. Me, N.T.B. Thuy, W. Siebel, T.G. Lan, Zircon U-Pb ages and $\mathrm{Hf}$ isotopic compositions from the Sin Quyen Formation: the Precambrian crustal evolution of northwest Vietnam, Int Geol Rev 54(2012) 15481561.https://doi.org/10.1080/00206814.2011.646831.

[31] T.T. Hoa, A.E. Izokh, G.V. Polyakov, A.S. Borisenko, T.T. Anh, P.A. Balykin, N.T. Phuong, S.N. Rudnev, V.V. Van, B.A. Nien, PermoTriassic magmatism and metallogeny of Northern Vietnam in relation to the Emeishan plume, Russ Geol Geophys 49(2008) 480-491. https://doi.org/ 10.1016/j.rgg.2008.06.005. 